\title{
LOS HIJOS DEL GUAYAS EN BUSCA DE LA LIBERTAD. ANÁLISIS HISTORIOGRÁFICO SOBRE EL PROTAGONISMO SOCIO-ÉTNICO EN LA INDEPENDENCIA DE GUAYAQUIL SEGÚN LOS TEXTOS ESCOLARES DE HISTORIA (1915-2015)
}

\section{THE SONS OF GUAYAS IN SEARCH OF FREEDOM. HISTORIOGRAPHIC ANALYSIS OF THE SOCIO- ETHNIC PROTAGONISM IN THE INDEPENDENCE OF GUAYAQUIL ACCORDING TO THE HISTORY TEXTBOOKS (1915-2015)}

\author{
Victor H. Silva Guijarro \\ Universidad Nacional de Educación a Distancia (UNED)
}

SUMARIO: I. INTRODUCCIÓN.- II. ANÁLISIS HISTORIOGRÁFICO DEL PROTAGONISMO SOCIO-ÉTNICO EN LA INDEPENDENCIA DE GUAYAQUIL SEGÚN LOS TEXTOS ESCOLARES DE HISTORIA.- 2.1. "Los Hijos del Guayas" versus el enemigo extranjero. Criollos y peninsulares como principales protagonistas de la Independencia (1915-1930).- 2.2. El pueblo, guardián de la República libre. Ascenso del protagonismo popular (19401990).- 2.3. Del olvido al abuso. Indígenas, negros y mestizos en la Independencia (2000-2015).- III. LOS GRANDES AUSENTES. ANÁLISIS SOBRE LA PARTICIPACIÓN DE LOS GRUPOS SOCIO-ÉTNICOS PRESENTADOS COMO VÍCTIMAS DEL SISTEMA COLONIAL.- IV. CONSIDERACIONES FINALES.

Resumen: El presente trabajo consiste en un análisis historiográfico sobre el protagonismo que tuvieron los distintos grupos socio-étnicos en la Independencia de Guayaquil según los textos escolares de Historia publicados entre 1915 y 2015. Se han utilizado como fuentes historiográficas los textos escolares de Historia, debido a que desde el siglo $\mathrm{XX}$ las politicas educativas ecuatorianas los han utilizado como instrumentos ideológicos para llevar a cabo la construcción de una identidad nacional común cimentada a partir de los presupuestos de la Historia Patria del siglo XIX. El empleo de la enseñanza de la Historia a través de los textos escolares para consolidar esa identidad nacional del siglo XIX se hace patente en el análisis sobre el protagonismo socio-étnico en la Independencia, donde dichos textos explican este acontecimiento como un proyecto de construcción nacional dirigido por las élites criollas cuyo supuesto objetivo principal era liberar a todos los "guayaquileños" de una presunta opresión monárquica ejercida por un enemigo extranjero: el "español". Pese a que hay autores que han manifestado que indígenas, negros, mestizos, zambos, pardos, mulatos, obtuvieron pocos beneficios de la Independencia, todos los textos escolares no dudan en confirmar que 
gracias a la gesta libertadora emprendida por las élites criollas de Guayaquil, el Ecuador pudo ser libre.

\begin{abstract}
The present work consists of a historiographic analysis of the protagonism that the different socio-ethnic groups had in the Independence of Guayaquil according to history textbooks published between 1915 and 2015. History textbooks have been used as historiographical sources, because since the 20th century Ecuadorian educational policies have used them as ideological instruments to carry out the construction of a common national identity based on the assumptions of the 19th century Homeland History. The use of history teaching through school textbooks to consolidate this 19th century national identity is evident in the analysis of the socioethnic protagonism in the Independence, where these texts explain this event as a national construction project led by the Creole elites whose supposed main objective was to liberate all "Guayaquileños" from a presumed monarchic oppression exercised by a foreign enemy: the "Spanish". Although there are authors who have stated that indigenous people, blacks, mestizos, zambos, pardos, mulatos, obtained few benefits from the Independence, all school textbooks do not hesitate to confirm that thanks to the liberating gesture undertaken by the Creole elites of Guayaquil, the Ecuador was able to be free.
\end{abstract}

Palabras clave: Textos escolares, Historia Patria, Protagonismo socio-étnico, Independencia de Guayaquil, Estado-nación, Identidad nacional.

Key Words: Textbooks, Homeland History, Socio-ethnic protagonism, Independence of Guayaquil, Nation State, National identity.

\title{
I. INTRODUCCIÓN
}

Desde el momento en el que los libertadores criollos obtuvieron la victoria en la Guerra de Independencia, el siguiente paso de los lideres de las nacientes repúblicas fue poner en marcha la construcción del Estado-nación como nuevo sistema político hegemónico. Este contexto obligó a los arquitectos de las nacientes patrias a crear y demostrar la existencia de la nación mediante la creación de una identidad nacional imaginada que llevara a cabo una diferenciación entre un "ellos" y un "nosotros". ${ }^{1}$ En el caso del Ecuador, tras su afianzamiento como República el principal objetivo de las élites dirigentes, representantes de una nación que habia que imaginarla, construirla y extenderla, ${ }^{2}$ fue el de arraigar y legitimar en el imaginario de los ecuatorianos el prototipo de nación y de identidad que querían construir mediante la consolidación de una personalidad nacional única y una trayectoria histórica común. Una de las herramientas empleadas para hacer realidad esta empresa fue la educación nacional, la

1 Tomás Pérez Vejo, Nación, identidad nacional y otros mitos nacionalistas, Ediciones Nobel, Oviedo, 1999, p. 44.

2 Tomás Pérez Vejo, España imaginada: historia de la invención de una nación, Galaxia Gutenberg, Barcelona, 2015, pp. 10-16. 
cual debía sustituir a la educación estamental del siglo XVIII. ${ }^{3}$ Dentro de esta educación, la enseñanza de la Historia Patria desempeñó un papel clave, ya que la cultura escolar del siglo XIX la utilizó para emprender esa construcción identitaria. ${ }^{4}$ Los textos escolares de Historia, como instrumentos idóneos para transmitir esos nuevos valores, cobran por tanto una singular importancia como fuentes para el estudio de la construcción nacional del país.

Desde el siglo XIX los Padres de la Patria comprendieron que el triunfo definitivo de su proyecto se encontraba en dar cuerpo a lo que Pérez Vejo ha definido como una construcción intelectual o mito de las élites; nos referimos a la nación. ${ }^{5}$ Es en este contexto donde los poderes politicos han utilizado la Historia Patria como una disciplina escolar para llevar a cabo una sintesis y transformación del proyecto de Estado-nación de las élites criollas en un objeto de enseñanza con la finalidad de insertar en los ciudadanos la conciencia de pertenencia a una nueva nación y generar un sentimiento de lealtad y confianza en las instituciones públicas. Esta situación no es producto del pasado puesto que los nacionalismos actuales siguen empleando la enseñanza de la historia por medio de los textos escolares para lograr no tanto la comprensión por parte de los alumnos de los problemas historiográficos, sino más bien inculcarles desde pequeños el "amor a la Patria" y el conocimiento de las glorias nacionales. ${ }^{6}$ Es por esta razón que dicha enseñanza ha sido uno de los sectores más disputados por los Estados-nación ya que su control garantiza ser árbitro y creador de la idea de nación. ${ }^{7}$

Este proyecto nacional del siglo XIX ha promovido una enseñanza de la Independencia de Guayaquil, presente en los textos escolares, a partir de una explicación imaginada e idealizada de este acontecimiento como un enfrentamiento entre dos naciones e identidades enfrentadas: "nosotros" los guayaquileños deseosos de libertad, versus "ellos" los españoles tiranos, que sirvió para cimentar las bases del Estado-nación de las élites criollas. Al ser conscientes de la gran importancia que tiene el texto escolar de Historia en la formación de la opinión pública ecuatoriana y en el desarrollo de la cultura nacional, ${ }^{8}$ asi como del protagonismo principal que ha desempeñado como elemento promotor de la identidad nacional de los Estados-nación surgidos tras la Independencia, se busca con este análisis colaborar en la

3 Gabriela Ossenbach, Formación de los sistemas educativos nacionales en Hispanoamérica. El caso ecuatoriano, 1895-1912, Corporación Editorial Nacional, Quito, 2018, pp. 65-71.

4 Carolyn P. Boyd, Historia Patria. Politica, historia e identidad nacional en España: 18751975, Ediciones Pomares-Corredor, Barcelona, 2000, p. 261. 12.

5 Tomás Pérez Vejo, España imaginada: historia de la invención de una nación, op. cit., p.

6 Mario Carretero, "La construcción de una identidad nacional", Cuadernos de Pedagogía, $\mathrm{n}^{\circ}$ 308, 2001, p. 53.

7 Paula de la Cruz Fernández, "Enseñando identidad nacional en el Ecuador", Minius: Revista de Departamento de Historia, Arte e Xeografia, n 16, 2008, p. 115.

8 Enrique Ayala Mora, Historiografia ecuatoriana: apuntes para una visión general, Corporación Editora Nacional, Quito, 2015, p. 132. 
puesta en valor del texto escolar no solo como un instrumento para llevar a cabo una nacionalización del pasado y del presente, sino también proponer su utilización como una herramienta que permita estudiar y analizar el pasado desde una perspectiva crítica. 9 Por esta razón, en este trabajo realizaremos un análisis historiográfico para conocer cual es el protagonismo otorgado por los textos escolares a los distintos grupos socio-étnicos en la Independencia de Guayaquil y contrastar el discurso sobre la emancipación, realizado por dichos textos escolares como una supuesta guerra anticolonial, con las investigaciones historiográficas académicas sobre este acontecimiento.

\section{ANÁLISIS HISTORIOGRÁFICO DEL PROTAGONISMO SOCIO-ÉTNICO EN LA INDEPENDENCIA DE GUAYAQUIL SEGÚN LOS TEXTOS ESCOLARES DE HISTORIA}

El corpus de esta investigación se articulará a partir de una pregunta: ¿cuál fue el protagonismo de los distintos grupos socio-étnicos en la Independencia de Guayaquil? Esta pregunta será utilizada como una herramienta para cumplir con todos los objetivos propuestos en esta investigación, los cuales consisten en conocer y analizar el protagonismo otorgado por los textos escolares a los diversos grupos socio-étnicos (peninsulares, criollos, mestizos, indigenas, negros, zambos, etc.) durante el desarrollo del proceso de escisión de la provincia guayaquileña. Para responder a esta cuestión se ha estudiado una muestra de quince textos escolares de enseñanza primaria y secundaria publicados entre $1915 \mathrm{y}$ 2015. Los resultados de esta investigación se presentarán en tres bloques cronológicos diferentes.

\section{1. "Los Hijos del Guayas" versus el enemigo extranjero. Criollos y peninsulares como principales protagonistas de la Independencia (1915- 1930)}

En el primer libro analizado de Bruño (1915), es llamativa la ausencia de datos sobre la Independencia de Guayaquil. En este breve resumen, el autor afirma que en 1820 Guayaquil proclamó su Independencia y se hizo cargo del gobierno el criollo José Joaquín de Olmedo. No obstante, esta empresa corría peligro ya que el General peninsular Melchor Aymerich envío tropas contra los patriotas criollos guayaquileños quienes fueron derrotados en Huachi y Tanizahua. Debido a los fracasos de los guayaquileños, entró en escena Simón Bolivar quien los auxilió enviando un contingente militar entre los que se encontraba Antonio José de Sucre. ${ }^{10} \mathrm{Si}$ en la obra anterior Bruño dedicó un apartado a Guayaquil, en el texto escolar de 1916 la explicación de la Independencia guayaquileña queda resumida como un acontecimiento que solo contó con la participación de los criollos venezolanos León Febres

\footnotetext{
${ }^{9}$ Raimundo Cuesta, "El código disciplinar de la Historia escolar en España: Algunas ideas para la explicación de la sociogénesis de una materia de enseñanza", Encounters in Theory and History of Education, Vol. 3, 2002, p. 36.

10 G. M. Bruño, Compendio de la Historia del Ecuador, Hermanos de las Escuelas Cristianas, Ecuador, 1915, pp. 60-62.
} 
Cordero y Luis Urdaneta. ${ }^{11}$ Cano (1920), se mantiene en la linea de Bruño afirmando que la Independencia de Guayaquil se logró gracias a los esfuerzos de Bolivar y Sucre. ${ }^{12}$ Tanto en los textos escolares de Bruño como en el de Cano, es destacada la ausencia de referencias sobre la participación de indigenas, negros, zambos, pardos, mulatos, etc., en la Independencia de lo que se desprende su exclusión de este acontecimiento.

Uzcátegui (1929), afirma que en un principio los guayaquileños de la época no buscaban la Independencia. Sin embargo, se sucedieron una serie de acontecimientos (Batalla de Boyacá, expedición de San Martín al Perú, etc.) que despertaron en los guayaquileños el deseo de emancipación. Según el autor, los encargados de realizar esta gesta fueron tres militares venezolanos: León Febres Cordero, Miguel Letamendi y Luis Urdaneta, quienes tuvieron una gran acogida entre los conspiradores de Guayaquil, algunos de los más destacados fueron: José María de Villamil, José María de Antepara, Juan Antonio Vivero, Francisco de Paula Lavayen, José Rivas, José Correa, Manuel Fajardo, entre otros. Uzcátegui, manifiesta que gracias a la acción conjunta de los criollos el 9 de octubre de 1820: "los españoles no volvieron a levantar cabeza en Guayaquil". ${ }^{13}$ Una vez consolidada la victoria, los patriotas nombraron Jefe Político del nuevo Estado al criollo guayaquileño José Joaquín de Olmedo, quien rápidamente convocó un Cabildo abierto para el nombramiento de una Junta de Gobierno que quedó presidida por José Joaquín de Olmedo, Rafael Jimena y Vicente Ramón Roca. A lo largo de toda la explicación sobre la Independencia guayaquileña, Uzcátegui no hace ninguna alusión sobre la participación de indigenas, negros, mulatos, zambos, etc. Simplemente realiza breves alusiones sobre el pueblo que aparece como protagonista en las celebraciones de la Independencia y como seguidores incondicionales de los libertadores. ${ }^{14}$

A diferencia de Bruño y Cano, Moscoso (1934) realiza un estudio más detallado sobre los protagonistas de la Independencia de Guayaquil. Este autor empieza su explicación del 9 de octubre de 1820, afirmando que tras las acciones libertadoras de Bolivar en Boyacá y San Martín en Maipú, "los hijos del Guayas" empezaron a prepararse para proclamar la libertad de la "Perla del Pacífico". Según Moscoso, los protagonistas de este acontecimiento fueron los criollos venezolanos Letamendi, Urdaneta y Febres Cordero, quienes lograron obtener el apoyo militar del Batallón Granaderos tras persuadir a Gregorio Escobedo (segundo jefe del batallón) y a los oficiales Hilario Álvarez y Dionicio Farfán. A estos militares se unieron los miembros más selectos del elemento civil de Guayaquil como José María de Villamil, Rafael Jimena, José Joaquín de Olmedo, entre otros. Los criollos guayaquileños lideres de la sociedad civil se adhirieron rápidamente a la

11 G. M. Bruño, Resúmenes de la Historia del Ecuador, Hermanos de las Escuelas Cristianas, Ecuador, 1916, pp. 47-48.

12 F.A. Cano, Nociones elementales de Historia del Ecuador, Prensa Católica, Ecuador, 1920, pp. 32-33.

13 Emilio Uzcátegui García, Historia del Ecuador. Texto para la Enseñanza de Historia Patria, Editorial Bolivar, Quito, 1929, p. 38.

14 Emilio Uzcátegui García, Historia del Ecuador. Texto para la Enseñanza de Historia Patria, op. cit., pp. 36-39. 
campaña militar de los venezolanos porque compartian las mismas ideas revolucionarias. ${ }^{15}$

Una vez conformado el grupo revolucionario de Guayaquil, Moscoso informa que en los choques militares que se produjeron el 9 de octubre de 1820, los actores principales que consumaron la victoria militar de Guayaquil contra la Corona fueron los criollos Febres Cordero, Urdaneta y Francisco de Paula Lavayen. Una situación similar se produjo en la organización del nuevo aparato político donde los principales cargos fueron ocupados por criollos. En un primer momento se nombró Jefe Político a José Joaquín de Olmedo y a Escobedo Jefe Militar. El 14 de octubre se formó una Junta de Gobierno compuesta por Gregorio Escobedo, Rafael Jimena y Vicente Espantoso, la cual se renovó el 8 de noviembre durante la reunión de la Asamblea donde se promulgó la $1^{\text {a }}$ Constitución y se nombró como Presidente a José Joaquín de Olmedo, Vocales a Francisco María Roca y Rafael Jimena, y Secretario a Francisco Marcos. Moscoso concluye su explicación de la Independencia de Guayaquil certificando que los únicos protagonistas que hicieron realidad la consolidación del Guayaquil independiente y libre fueron los criollos. Por último, realiza una breve mención sobre la participación del "pueblo" y el papel que desempeña es el de un conglomerado que rinde honor a los jóvenes héroes y libertadores de Guayaquil. ${ }^{16}$

Analizados todos los textos escolares de este primer bloque cronológico, la idea que podemos sacar en claro sobre el protagonismo de la Independencia de Guayaquil es que fue un acontecimiento político y bélico que tuvo como únicos protagonistas a criollos $\mathrm{y}$ peninsulares. Para corroborar esta tesis hemos realizado un recuento del número de veces en el que los distintos grupos socio-étnicos son partícipes de los acontecimientos ocurridos en Guayaquil según los textos escolares publicados entre 1915 y 1930. Los resultados obtenidos (gráfica 1) están marcados por el predominio de criollos y peninsulares. Hay breves alusiones al pueblo en general, pero su protagonismo se reduce a cumplir un rol de "pueblo salvado" gracias a la gesta emprendida por los libertadores. Debemos informar que ninguno de los autores habla de la participación de mestizos, negros, indígenas, zambos, pardos, mulatos, etc., en el proceso emancipatorio de Guayaquil. Por último, cabe destacar que tanto Uzcátegui como Moscoso realizan una explicación de la Revolución de Guayaquil como una de las etapas decisivas de la Independencia ecuatoriana porque, según los autores, en el proyecto libertador de los criollos guayaquileños se encontraba el deseo de liberar a los demás pueblos de la Sierra y lograr la definitiva Independencia del Ecuador.

15 Leonardo Moscoso, Lecciones Elementales de la Historia General del Ecuador, J. Roberto Cruz, Quito, 1934, pp. 77-78.

16 Leonardo Moscoso, Lecciones Elementales de la Historia General del Ecuador, op.cit., pp. 77-78. 


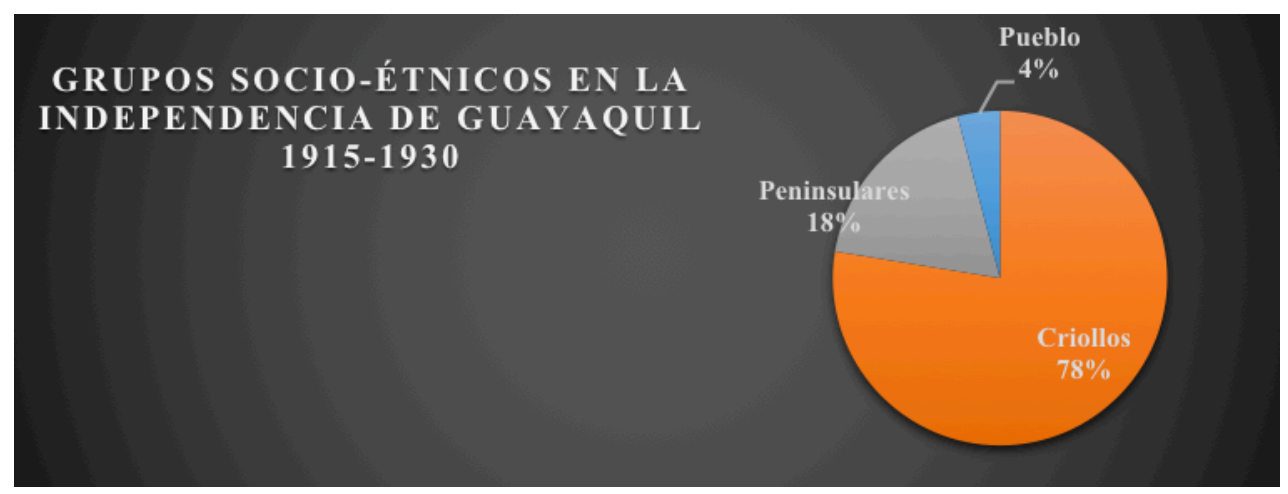

Gráfica 1. Análisis del protagonismo socio-étnico en la Independencia de Guayaquil (19151930). Fuente: Elaboración propia a partir de Bruño, 1915-1916; Cano, 1920; Uzcátegui, 1929; Moscoso, 1934.

\subsection{E1 pueblo, guardián de la República libre. Ascenso del protagonismo popular (1940-1990)}

Cevallos (1972), inicia su explicación de la Revolución de Guayaquil dedicando un apartado exclusivo para estudiar a Melchor Aymerich, presidente de la Audiencia de Quito, porque fue durante su mandato cuando surgió en Guayaquil el deseo de independizarse. El autor aporta un dato de especial interés afirmando que para las autoridades peninsulares Guayaquil no suponía una preocupación porque siempre fue considerada como un centro monárquico. No obstante, la ciudad empezó a ser vigilada por los realistas borbónicos porque en el seno de la sociedad guayaquileña empezaron a obtener un protagonismo cada vez más creciente un grupo de criollos definidos como "republicanos guayaquileños". Ante el temor a una posible extensión de ideas republicanas, Aymerich ordenó resguardar la ciudad enviando un gran contingente militar. Sin embargo, esto no supuso un problema para los criollos de Guayaquil quienes aprovecharon las fiestas sociales para organizar la conspiración contra la Corona. Según el autor, los principales animadores de la Independencia de Guayaquil fueron esencialmente tres: Febres Cordero, Letamendi y Urdaneta, los cuales son definidos como oficiales republicanos con una gran capacidad de persuasión. A estos se sumaron los jóvenes más notables de Guayaquil como José María de Antepara, Lorenzo Garaicoa, Francisco de Paula Lavayen, Vicente Ramón Roca y José Vallejo, entre otros. Una vez convencidos para hacer la revolución, Cevallos plantea que los protagonistas se precipitaron en la toma de decisiones poniendo en peligro la Independencia debido a que "un grupo de personas tímidas" denunciaron al gobernador de Guayaquil sobre los planes revolucionarios. Las fuerzas realistas se extendieron por la ciudad para frenar a los conspiradores, pero esta reacción no pasó a mayores ya que las autoridades no tenian pruebas veraces sobre el golpe que se preparaba. Esta situación llevó a los protagonistas de la conspiración a adelantar el golpe contra los realistas y el 9 de octubre "Guayaquil pasó al régimen republicano [...] y permaneció libre e invicta desde el día de su Independencia". ${ }^{17}$

17 Gabriel Cevallos García, Historia del Ecuador $5^{\circ}$ Curso. Ciclo Diversificado, Don Bosco, Cuenca, 1972, pp. 256-259. 
Una vez consolidada la victoria militar, Cevallos afirma que los vencedores entregaron el poder del mando civil a José Joaquín de Olmedo, quien convocó una "gran reunión popular para que la nueva realidad politica tomara forma legal republicana". ${ }^{18}$ En Guayaquil se estableció una Junta Gubernativa popular compuesta por Escobedo, Espantoso, Jimena y Vivero, que llevó a cabo la convocatoria de un Colegio Electoral y el 8 de noviembre se erigió la nueva Junta Suprema precedida por Olmedo, Jimena y Roca, y se redactó la Constitución de Guayaquil. Cevallos se mantiene en la línea de los anteriores autores afirmando que la revolución se pensó, desarrolló y construyó en torno a un único núcleo de protagonistas; los criollos de Guayaquil, donde el "pueblo" solo aparecía para las celebraciones de las victorias. Aunque también hace una lectura diferente sobre ese protagonismo de la acción popular planteando que, una vez consolidada la Independencia, el pueblo tuvo que esforzarse por mantener la libertad de la nueva República. ${ }^{19}$

Cevallos en su libro publicado en 1978 realiza una explicación sobre los protagonistas de la Independencia de Guayaquil similar al texto escolar de 1972, aunque aportando nueva información. Del mismo modo que en el anterior, otorga un protagonismo central a los militares venezolanos (Febres Cordero, Letamendi y Urdaneta) ya que en torno a ellos se generó la conspiración contra la Corona. Los criollos que antes fueron definidos como "jóvenes notables", ahora el autor se refiere a ellos como "patriotas". De este selecto grupo, Cevallos destaca a José de Villamil, Antepara, Lavayen y a otros patriotas de Guayaquil que no han sido mencionados hasta ahora como a Luis Fernando de Vivero y Antonio Elizalde. Afirma el autor que después de convencer a los jefes militares de las tropas, Villamil y Antepara fueron los encargados de preparar la primera reunión de los cabecillas del movimiento. Dicha reunión se celebró la noche del 1 de octubre de 1820 utilizando como pretexto la organización del baile de honor de la hija del Ministro de las Cajas Reales; Pedro Morlás. Estas reuniones se extendieron hasta el 5 de octubre buscando al candidato ideal para que ocupara el cargo de Jefe Político de la revolución. Villamil propuso al Coronel Jacinto Bejarano, al Doctor José Joaquín de Olmedo y al Teniente Coronel Rafael Jimena, pero todos declinaron tal propuesta. ${ }^{20}$

Convencido el mando político y repartidas las misiones que consolidarian la revolución, el 9 de octubre de 1820 los patriotas lograron la victoria. Otro dato novedoso que podemos encontrar en esta obra consiste en que una vez emitida el acta de Independencia, el Jefe Politico (Olmedo) recibió del pueblo y de las tropas el juramento a ser "independientes, fieles a la Patria y defenderla con todas las fuerzas que estén a sus alcances".21 Asimismo, los líderes criollos propusieron a los empleados de los antiguos 259.

18 Gabriel Cevallos García, Historia del Ecuador $5^{\circ}$ Curso. Ciclo Diversificado, op. cit., p.

19 Gabriel Cevallos García, Historia del Ecuador $5^{\circ}$ Curso. Ciclo Diversificado, op. cit., pp. 259-260.

20 Gabriel Cevallos García, Historia del Ecuador $5^{\circ}$ Curso. Colección LNS, Don Bosco, Cuenca, 1978, p. 221.

${ }^{21}$ Gabriel Cevallos García, Historia del Ecuador $5^{\circ}$ Curso. Colección LNS, op. cit., p. 221. 
ministerios seguir con sus servicios siempre y cuando juren ser independientes y fieles a la Patria, y se comprometan a luchar por la libertad de América. La explicación sobre los protagonistas de los acontecimientos posteriores es semejante al texto escolar anterior, es decir, la Junta Provisional de gobierno se encargó de convocar un Colegio Electoral para elegir como Presidente a Olmedo, Vocal de asuntos militares a Rafael Jimena, Vocal de Asuntos político-civiles a Francisco M. Roca y Secretario a Francisco Marcos. De esta manera, quedó consolidado el nuevo gobierno criollo y republicano, el cual emitió el 8 de noviembre de $1820 \mathrm{su}$ primera Constitución. ${ }^{22}$

Carrillo (1997), realiza una explicación de la Independencia de Guayaquil de una forma concreta $y$ no aportando tantos datos historiográficos. La autora afirma de forma contundente que la etapa final y decisiva de la Independencia del Ecuador se inició en Guayaquil gracias a la gesta libertadora emprendida por los criollos de esta provincia como Villamil, Elizalde, Antepara, Garaicoa, Vivero, etc., y por los criollos venezolanos como Febres Cordero, Letamendi y Urdaneta. Estos y otros patriotas al grito de ¡Viva la Patria! depusieron a las autoridades españolas y consolidaron la Independencia de Guayaquil el 9 de octubre de 1820. Posteriormente, la provincia guayaquileña promulgó su Carta Fundamental y se entregó el poder al triunvirato compuesto por Olmedo, Jimena y Roca. ${ }^{23}$

Núñez (1997), también realiza una explicación de la Independencia de Guayaquil sintetizada y estructurada en torno a cuatro ideas que dan un giro total a la interpretación realizada hasta ahora por los textos escolares sobre los protagonistas de este acontecimiento. El autor parte informando que la reacción antimonárquica surgió en Guayaquil debido a las prácticas monopólicas en el negocio del cacao de los comerciantes del Consulado de Lima, quienes extorsionaron fiscalmente a los comerciantes y propietarios guayaquileños para financiar la guerra del Virreinato del Perú contra el ejército libertador de San Martín. A esta situación se sumó la fundación de una logia masónica guayaquileña llamada "La Fragua de Vulcano" donde empezó la conspiración anticolonial. Esta organización estaba compuesta en un principio por la élite criolla de Guayaquil a la que posteriormente se sumaron los capitanes venezolanos (Urdaneta, Letamendi y Febres Cordero), quienes juntos llevaron a cabo la insurrección libertadora el 9 de octubre de 1820. Consolidada la Independencia, el autor deja entrever que los criollos de Guayaquil tuvieron la responsabilidad de liberar a toda la Patria y es por esta razón que la nueva Junta Gubernativa se propuso como objetivo principal independizar a la Sierra. Finalmente, Núñez en su análisis de la Independencia de Guayaquil propone que este acontecimiento debería ser estudiado como una guerra civil debido a que tanto el ejército realista como el insurgente contó en sus filas con la presencia de criollos y peninsulares. Pese a las novedosas ideas propuestas en este texto escolar, nuevamente no 222 .

22 Gabriel Cevallos García, Historia del Ecuador $5^{\circ}$ Curso. Colección LNS, op. cit., pp. 221-

23 Rogelia Carrillo de Landázuri, El libro del Escolar Ecuatoriano 6, Edimpres, Quito, 1997, pp. 350-351. 
encontramos ninguna alusión sobre la participación de indígenas, negros, zambos, pardos, mulatos y mestizos en la Independencia. ${ }^{24}$

Al realizar el recuento de los protagonistas de la Independencia según los textos escolares de este bloque cronológico (gráfica 2), encontramos algunos cambios con respecto al bloque anterior. Es destacado el aumento de la participación del pueblo, que sigue cumpliendo su rol de "pueblo salvado" gracias a la magnífica obra de los criollos. Aunque hay un autor, Cevallos, que otorga un protagonismo diferente al pueblo, el cual, tenía que cumplir la función de guardián de la nueva República defendiendo la libertad lograda por los próceres criollos. ${ }^{25}$ Todos los autores de este bloque cronológico no dudan en consolidar la idea de que Guayaquil logró su Independencia gracias a la élite criolla que se enfrentó a los peninsulares para obtener la libertad del pueblo guayaquileño. ${ }^{26}$ Con respecto a la participación de indigenas, negros, zambos, pardos, mulatos, mestizos, etc., en la Independencia de Guayaquil, los datos que nos aportan los autores de este bloque cronológico son contundentes y todos se reafirman en la idea de que este acontecimiento tuvo como únicos protagonistas a criollos $\mathrm{y}$ peninsulares. Tanto Cevallos como Carrillo no aportan una explicación sobre esta situación, en cambio Núñez afirma tajantemente que la Independencia de Guayaquil no contó con la participación de indígenas, negros, mestizos, etc., porque fue una guerra civil. No obstante, Núñez no desarrolla en profundidad esta propuesta teórica, habla de ella como una posibilidad que, desde nuestro punto de vista, tendrá que ser discernida por el lector.

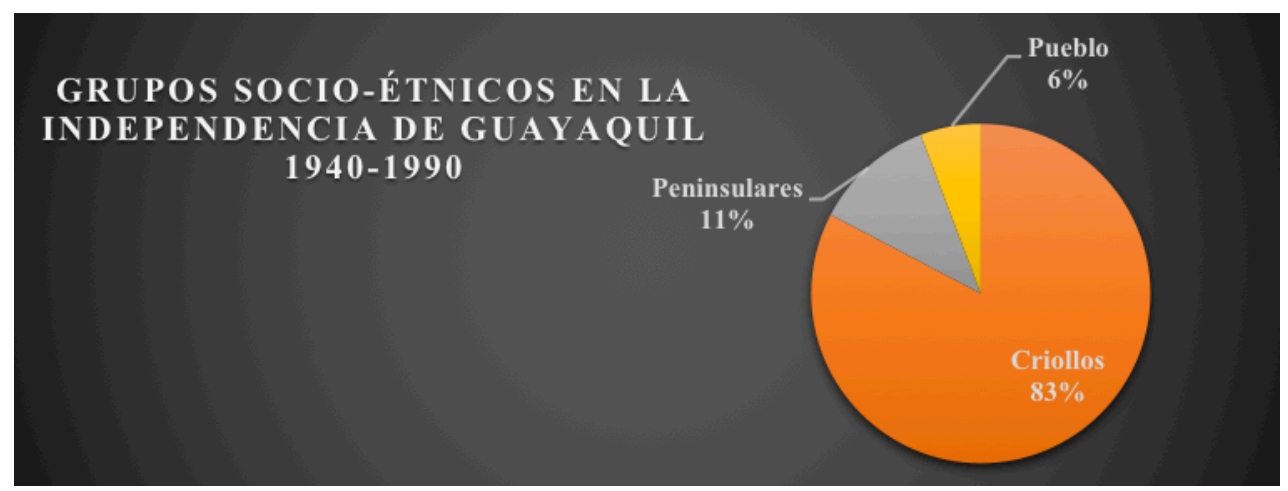

Gráfica 2. Análisis del protagonismo socio-étnico en la Independencia de Guayaquil (19401990). Fuente: Elaboración propia a partir de Cevallos, 1972-1978; Carrillo,1997; Núñez, 1997.

\subsection{Del olvido al abuso. Indigenas, negros $y$ mestizos en la Independencia (2000-2015)}

A diferencia de los textos escolares editados por Uzcátegui, Moscoso y Cevallos, los autores de las obras que analizaremos para este periodo no realizan exposiciones tan detalladas sobre la Independencia de Guayaquil.

\footnotetext{
${ }^{24}$ Jorge Núñez Sánchez, Historia del Ecuador, Santillana, Quito, 1997, p. 10.

25 Gabriel Cevallos García, Historia del Ecuador $5^{\circ}$ Curso. Ciclo Diversificado, op. cit., pp. 256-260.

${ }^{26}$ Rogelia Carrillo de Landázuri, El libro del Escolar Ecuatoriano 6, op. cit., p. 350.
} 
Por esta razón, con el objetivo de que la lectura no se vuelva repetitiva, solo explicaremos aquellos detalles novedosos que nos permitan conocer a nuevos protagonistas y los planteamientos que realizan los autores sobre el protagonismo socio-étnico en la Independencia.

En el texto escolar de Navas (2001), no encontramos cambios significativos. Este autor destaca como principales protagonistas de la Independencia de Guayaquil a los militares venezolanos y a los patriotas guayaquileños como Isidoro Viteri, Diego Noboa, Lorenzo Garaicoa, José María de Antepara, etc. No obstante, Navas relata el desarrollo de un acontecimiento clave para la consolidación de la Independencia que nos permite conocer a otros protagonistas de este acontecimiento como al Teniente Damián Nájera, quien en la noche del 9 de octubre con la excusa de jugar a los naipes invitó a su casa al Teniente Coronel de las fuerzas realistas Manuel Torres Valdivia, el cual, fue arrestado por Nájera y Febres Cordero. Una vez neutralizado el líder del ejército realista, Febres Cordero y Escobedo tomaron el cuartel de Artilleria y Luis Urdaneta junto a varios jóvenes guayaquileños tomaron el cuartel Daule asesinando al Comandante español Joaquín Magallar. De esta manera, los patriotas vencieron a los realistas y proclamaron la Independencia de Guayaquil cuyo gobierno quedó presidido por el ya mencionado triunvirato (Olmedo, Jimena y Roca). Navas plantea que la libertad definitiva de Guayaquil con respecto a España se consiguió gracias a los criollos que formaban parte de las familias distinguidas de Guayaquil. El autor concluye afirmando que en esta lucha por la libertad de Guayaquil no se contó con la participación de indígenas, negros, zambos, pardos, mulatos o mestizos, porque fue un acontecimiento que tuvo como únicos protagonistas a criollos versus peninsulares. ${ }^{27}$

Holguín (2003), en su estudio sobre la Independencia incorpora la situación colonial. Es por este motivo que en su obra se visibiliza un aumento del protagonismo de los grupos socio-étnicos marginados (indigenas, negros, zambos, etc.), los cuales aparecen representados como los principales articuladores de los levantamientos populares durante el periodo colonial. Este texto escolar se desmarca de lo planteado hasta el momento por los anteriores autores, ya que Holguín otorga un mayor protagonismo durante la Independencia a los indigenas, negros y mestizos no como grupo dirigente, sino como víctimas de todo el periodo colonial. Concretamente sobre la Independencia de Guayaquil, el autor presenta a nuevos protagonistas del proceso revolucionario como a los principales firmantes del Acta de Independencia: José Joaquín de Olmedo, Manuel de José Herrera, Gabriel García Gómez, José Antonio Espantoso, Pedro Santander, José M. Maldonado, José Ramón Menendez, Francisco Marcos y José de Villamil. Una vez lograda la Independencia, Holguín plantea que los próceres guayaquileños organizados en torno al Primer Cuerpo Legislativo otorgaron "al país una Primera Constitución". Este autor se refiere a Guayaquil como "pais" porque considera que el movimiento revolucionario del 9 de octubre, protagonizado por los criollos guayaquileños y venezolanos, influyó en todos los movimientos libertadores que se dieron por el Ecuador

27 Mario Navas Jiménez, Estudios Sociales 9, Quebecor World Bogotá, Quito, 2001, pp. 128-129. 
hasta llegar a la Batalla del Pichincha el 24 de mayo de 1822. Holguín concluye afirmando que los protagonistas de este acontecimiento idearon un proyecto real de Independencia que buscaba la consolidación de una identidad nacional y cultural, y la independencia económica y política. Por esta razón, insiste en que esta revolución ideada y pensada por la élite criolla de Guayaquil debe ser estudiada como un proyecto anticolonial. ${ }^{28}$

Muñoz (2007), consolida la idea de que los principales actores de la Independencia fueron los representantes de los grupos étnicos humillados y explotados, es decir los mestizos y los indigenas, el deseo de libertad surgió en estos porque fueron las víctimas del dominio español. Afirma el autor, que debido al ultraje y desprecio que sufrieron los mestizos al considerarlos como no aptos para desempeñar los cargos que ocupaban los peninsulares, los precursores del sentimiento independentista fueron los mestizos con formación. No obstante, pese a este novedoso protagonismo otorgado a los mestizos, en esta obra nuevamente se vuelve a certificar que los protagonistas de la Independencia de Guayaquil fueron los criollos, quienes se convirtieron en los abanderados de la lucha por la libertad contra los peninsulares. Muñoz destaca que los principales gestores de la Independencia de Guayaquil fueron José de Villamil porque puso todos los medios materiales y su influencia como Procurador de la ciudad, y León Febres Cordero porque fue el alma de la revolución. En torno al militar venezolano se adhirieron Luis Fernando de Vivero, Francisco de Paula Lavayen, Agustín Lavayen, Miguel Lavayen, Manuel Lavayen, Lorenzo Garaicoa, Guillermo Bodero, Francisco Marcos, Francisco de Elizalde, Antonio de Elizalde y otros muchos patriotas encargados de gestar la revolución. Una vez lograda la victoria contra los peninsulares, la tarea de crear la nueva forma de gobierno tuvo como principal protagonista a José Joaquín de Olmedo, quien como Jefe Político convocó una Asamblea de Representantes de toda la provincia de Guayaquil con el objetivo de que ella decida la mejor forma de gobierno. Como ya hemos anunciado, el 8 de noviembre se reunió la asamblea donde se emitió la primera Carta Política y se designó una nueva Junta de Gobierno compuesta por Olmedo, Jimena, Roca y Marcos. ${ }^{29}$

En el texto escolar editado por el Ministerio de Educación (2008), se plantea que en la Independencia de Guayaquil los patriotas guayaquileños decidieron poner fin al gobierno extranjero porque su economía se vio afectada por el monopolio comercial y por las restricciones y prohibiciones que ejercian los españoles. En esta obra se destaca como principales protagonistas de la revolución a los guayaquileños José Joaquín de Olmedo y Vicente Rocafuerte, gente ilustrada que conocian la Constitución de Cádiz. A estos se unieron en octubre de 1820 los militares venezolanos y el grupo de guayaquileños liderados por Villamil y Antepara, quienes el 9 de octubre de 1820 declararon la Independencia de Guayaquil y abrieron el camino definitivo a la liberación del Ecuador el 24 de mayo de 1822. En este texto escolar no se realiza ninguna alusión sobre la participación de indigenas, negros, zambos, mulatos, etc., en la Revolución de Guayaquil. Sus únicos

28 Rubén Holguin Arias, Estudios Sociales 5, Holguin Ediciones, Quito, 2003, pp. 75-77.

29 Hernán Muñoz, Estudios Sociales, Prolipa, Quito, 2007, pp. 112-128. 
protagonistas fueron los criollos guayaquileños y venezolanos quienes derrotaron a los peninsulares. ${ }^{30}$

Ayala Mora (2010), realiza una explicación sobre los protagonistas de la Independencia de Guayaquil manteniéndose en la línea de los anteriores autores. Destaca la labor emprendida por los militares venezolanos y los notables guayaquileños quienes resolvieron declarar la Independencia el 9 de octubre de 1820. Por encima de todos los protagonistas, el autor realza la labor emprendida por José Joaquín de Olmedo quien es definido como la gran figura del pronunciamiento. Al igual que en los anteriores textos escolares, no se realiza ninguna mención sobre la participación de indígenas o negros durante este acontecimiento. El autor plantea que los principales protagonistas de la Independencia fueron los criollos latifundistas poseedores de títulos de marqueses y condes y los criollos activistas, abogados, intelectuales, medianos terratenientes y sacerdotes. Asimismo, considera que los grupos populares urbanos y campesinos mestizos no apoyaron en un principio la causa revolucionaria, aunque posteriormente se incorporaron a la rebelión anticolonial convirtiéndose en el grueso de las tropas de guerra. Con respecto a los indígenas, el autor afirma que su escasa participación se debe a que no apoyaron la causa independentista porque no les favorecía, e incluso hubo algunos grupos que apoyaron al ejército realista. Todo lo contrario ocurrió con la comunidad negra, la cual, según el autor, al entender que su participación en la guerra era una vía de liberación de la esclavitud y de ascenso social se alistaron al ejército patriótico. ${ }^{31}$

Debido a que en el texto escolar de De la Cruz y Ruano Gómez (2015) se realiza un estudio de la Independencia centrado principalmente en el análisis de aquellas teorias politicas que hicieron posible la revolución, solo encontramos breves alusiones sobre los protagonistas de este acontecimiento. Los autores resumen el estudio del protagonismo socioétnico de la Independencia afirmando que debido al deseo de los criollos por lograr la emancipación estos se convirtieron en los abanderados de la lucha contra la liberación de España. ${ }^{32}$

Como se puede apreciar en el recuento de la participación de los diferentes grupos socio-étnicos en la Independencia (gráfica 3), aparecen por primera vez en escena nuevos grupos como los indígenas, negros y mestizos y esto se debe a que autores como Muñoz y Holguín les otorgan un mayor protagonismo al definirlos como víctimas del periodo colonial. En la obra de Ayala Mora encontramos una lectura diferente sobre el aumento del protagonismo de estos grupos socio-étnicos. Este autor es el único que se refiere a los indigenas, negros y mestizos como actores activos de la Independencia, pero no como dirigentes de la campaña libertadora, sino como miembros de las infanterías de guerra tanto del ejército realista como del patriota. Por último, la explicación sobre el gran protagonismo otorgado a

30 Ministerio de Educación, Estudios Sociales 9, Gobierno de Pichincha, Pichincha, 2008, pp. 88-94.

${ }^{31}$ Enrique Ayala Mora, Estudios Sociales 6. Historia del Ecuador I. Época Aborigen, Colonia e Independencia, Corporación Editora Nacional, Quito, 2010, pp. 84-87.

32 Katherine de la Cruz y Olver Ruano Gómez, Enigmas I. Historia y Ciencias Sociales, Argo, Guayaquil, 2015, p. 188. 
los criollos en estos textos escolares se debe a que este acontecimiento es estudiado como un proyecto anticolonial, donde las élites criollas guayaquileñas se enfrentaron a los españoles para acabar con el sistema colonial y crear una nueva sociedad en torno a una supuesta República libre.

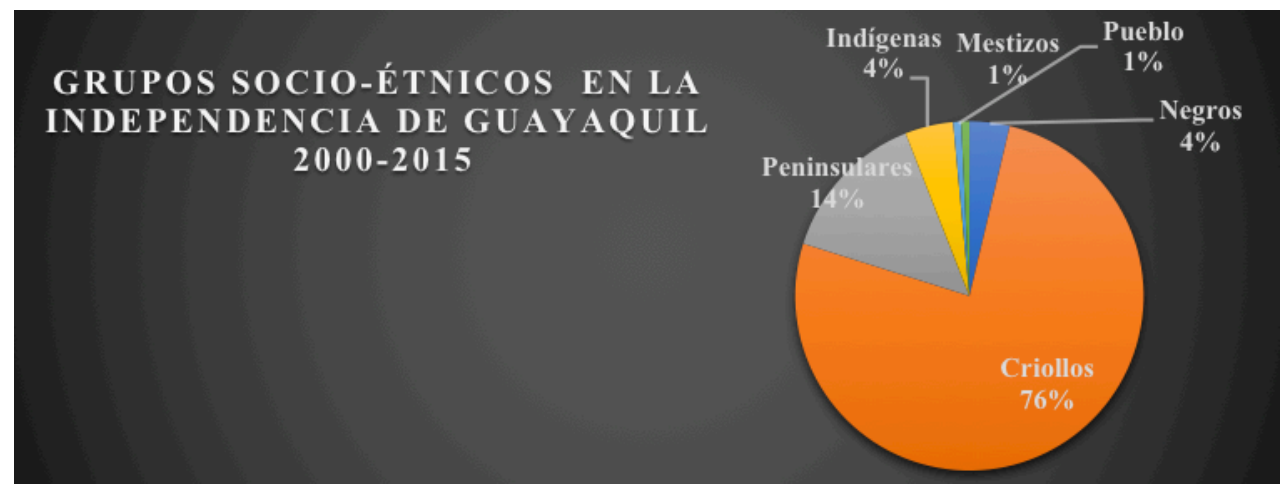

Gráfica 3. Análisis del protagonismo socio-étnico en la Independencia de Guayaquil (20002015). Fuente: Elaboración propia a partir de Navas, 2001; Holguín, 2003; Muñoz, 2007; Ministerio de Educación, 2008; Ayala Mora, 2010; De la Cruz y Ruano Gómez, 2015.

\section{LOS GRANDES AUSENTES. ANÁLISIS SOBRE LA PARTICIPACIÓN DE LOS GRUPOS SOCIO-ÉTNICOS PRESENTADOS COMO VÍCTIMAS DEL SISTEMA COLONIAL}

A lo largo de esta investigación hemos podido apreciar que todos los textos escolares analizados presentan una misma hipótesis sobre el protagonismo en la Revolución de Octubre, afirmando que este fue un acontecimiento que tuvo como principales protagonistas a los próceres criollos de Guayaquil (tabla 1) quienes se enfrentaron a los españoles representantes de un gobierno extranjero. ${ }^{33}$ Los textos escolares publicados en el siglo XX afirman de manera contundente que el protagonismo de indígenas, negros, zambos, pardos, mestizos, etc., en la Independencia de Guayaquil fue nulo (véase gráfica 1 y 2). Esta dinámica cambia en los textos escolares del bloque correspondiente al XXI donde se puede apreciar la participación, aunque mínima, de indígenas, negros y mestizos. El aumento de dicha participación se debe a que hay dos autores (Holguín y Muñoz) que otorgan un mayor protagonismo a indígenas y negros, aunque este protagonismo radica en su condición de víctimas de la opresión del sistema colonial y no como héroes de la gesta independentista. Como hemos podido apreciar, estos dos autores aprovechan sus obras para denunciar que desde la consolidación del sistema colonial tanto el indígena como el negro han arrastrado una condición de víctimas y esclavos. Tomando como punto de partida investigaciones historiográficas actualizadas debemos afirmar que, en contraposición a lo propuesto por los textos escolares, la realidad del indígena en el periodo colonial no se basó exclusivamente en una relación con los peninsulares articulada a partir de la dualidad vencedor/vencido,

${ }^{33}$ Ministerio de Educación, Estudios Sociales 9, op. cit., p. 88. 
explotador/explotado. ${ }^{34}$ Cabe recordar que a lo largo del siglo XVII y principios del XVIII, la sociedad indigena representada por la República de Indios disfrutaba de una amplia permisibilidad, ${ }^{35}$ tenían derecho a poseer sus propias lenguas, culturas, leyes, tradiciones y tierras. ${ }^{36}$

En contraposición a lo propuesto por los textos escolares, el indígena durante el periodo colonial fue un actor activo. Bien es cierto que la Conquista alteró su realidad, pero esto no supuso un inmovilismo, todo lo contrario, se adaptó y luchó para alcanzar sus objetivos y en algunos casos (con el apoyo de las autoridades coloniales) obtener un gran poder que ponía en peligro a los intereses y negocios de las propias autoridades peninsulares. A modo de ejemplo, Jaime Rodríguez en su estudio sobre la Independencia de Guayaquil plantea que en la sociedad guayaquileña del siglo XVIII y principios del XIX, debido a la diversidad de población y economía se generó uno de los ambientes más igualitarios del Antiguo Régimen. Afirma este autor, que las oportunidades económicas que se daban en la provincia promovieron la movilidad social y la posibilidad de que "los miembros de todos los grupos étnicos, incluidos los de ascendencia africana, se convirtieran en prominentes miembros de la sociedad". ${ }^{37}$ De esta manera, podemos plantear que, en contraposición a lo propuesto por los textos escolares, tanto el indígena como el negro también tuvieron la oportunidad de ser agentes activos de la sociedad del periodo colonial. Rodriguez afirma que la participación de indígenas, negros, mestizos, etc., fue notoria en los cambios sociales que trajo consigo la Constitución de Cádiz en la provincia de Guayaquil como ocurrió, por ejemplo, en las elecciones de funcionarios para el nuevo Ayuntamiento Constitucional de Guayaquil en 1813. En este proceso electoral se atestigua la participación de indigenas, mestizos e incluso de negros y mulatos como electores y en algunos casos como candidatos. Por ejemplo, en pueblos de Daule, Jipijapa y Montecristi, se eligieron como representantes politicos a indigenas, mulatos y mestizos, los cuales buscaban imponer su autoridad. ${ }^{38}$

34 María Eugenia Chávez, "Los sectores subalternos y la retorica libertaria. Esclavitud e inferioridad racial en la gesta independentista”, en Guillermo Bustos y Armando Martínez (edit.), La Independencia en los Paises Andinos: Nuevas perspectivas, Universidad Andina Simón Bolivar / Organización de Estados Iberoamericanos, Bucaramanga, 2004, pp. 209218 .

35 Jaime Rodríguez Ordóñez, "La Revolución de la Independencia Hispanoamericana frente a las Revoluciones Atlánticas. Perspectivas comparativas”, en Guillermo Bustos y Armando Martínez (edit.), La Independencia en los Paises Andinos: Nuevas perspectivas, Universidad Andina Simón Bolivar / Organización de Estados Iberoamericanos, Bucaramanga, 2004, pp. 18-19.

36 Horts Pietschmann, "Las élites politicas de México en la época de la emancipación, 1770 1830", en Luis Táuregui y José Antonio Serrano, (edit.), Historia y nación, El Colegio de México, México, 1998, p. 58.

37 Jaime Rodríguez Ordóñez, "De la fidelidad a la revolución: el proceso de la Independencia de la antigua provincia de Guayaquil, 1809-1820", Procesos. Revista Ecuatoriana de Historia, $n^{\circ} 21,2004$, pp. 38-45.

38 Jaime Rodríguez Ordóñez, "De la fidelidad a la revolución: el proceso de la Independencia de la antigua provincia de Guayaquil, 1809-1820”, op. cit., pp. 67-77. 


\begin{tabular}{|c|c|c|}
\hline \multicolumn{3}{|c|}{ PRÓCERES DE LA INDEPENDENCIA DE GUAYAQUIL } \\
\hline CRIOLLOS & $\begin{array}{l}\text { José Maria de Antepara } \\
\text { Jacinto Bejarano } \\
\text { Guillermo Bodero } \\
\text { José Correa } \\
\text { Antonio de Elizalde } \\
\text { Francisco de Elizalde } \\
\text { Gregorio Escobedo } \\
\text { José Antonio Espantoso } \\
\text { Vicente Espantoso } \\
\text { Manuel Fajardo } \\
\text { Dionicio Farfán } \\
\text { León Febres Cordero } \\
\text { Luis Fernando de Vivero } \\
\text { Lorenzo Garaicoa } \\
\text { García Gómez } \\
\text { Manuel de José Herrera } \\
\text { Rafael Jimena } \\
\text { Francisco de Paula Lavayen }\end{array}$ & $\begin{array}{l}\text { Agustín Lavayen } \\
\text { Manuel Lavayen } \\
\text { Miguel Lavayen } \\
\text { Miguel Letamendi } \\
\text { José M. Maldonado } \\
\text { Francisco Marcos } \\
\text { José Ramón } \\
\text { Menendez Maria } \\
\text { Damián Nájera } \\
\text { Diego Noboa } \\
\text { José Joaquín de } \\
\text { Olmedo } \\
\text { José Rivas } \\
\text { Francisco } \\
\text { Roca } \\
\text { Vicente Ramón Roca } \\
\text { Pedro Santander } \\
\text { Luis Urdaneta } \\
\text { José Vallejo } \\
\text { José Maria de } \\
\text { Villamil } \\
\text { Isidoro Viteri } \\
\text { Juan Antonio Vivero }\end{array}$ \\
\hline PENINSULARES & Pedro Morlás & \\
\hline
\end{tabular}

Tabla 1. Próceres de la Independencia de Guayaquil. Fuente: Elaboración propia a partir de Bruño, 1915-1916; Cano, 1920; Uzcátegui, 1929; Moscoso, 1934; Cevallos, 1972-1978; Carrillo,1997; Núñez, 1997; Navas, 2001; Holguín, 2003; Muñoz, 2007; Ministerio de Educación, 2008; Ayala Mora, 2010; De la Cruz y Ruano Gómez, 2015.

\section{IV-. CONSIDERACIONES FINALES}

El uso público de la Historia en el proceso de creación y de imaginación de identidades sociales, politicas y culturales ha estado unido al nacimiento de los Estados-nación. ${ }^{39}$ Es por esta razón que desde el siglo XIX hasta la actualidad, la enseñanza del proceso de Independencia en el Ecuador ha estado sujeta a los objetivos politicos establecidos por las reformas curriculares de los diferentes gobiernos, y las editoriales que publican los textos escolares deben acogerse a lo estipulado por las leyes educativas en lo referente al estudio de la Independencia. Esta situación ha provocado que los textos escolares hayan sido utilizados como instrumentos ideológicos para construir una identidad nacional, mostrando continuidad en una explicación de la Independencia ecuatoriana que se gestó en los planteamientos de la Historia Patria surgida en el siglo XIX.

La utilización de la enseñanza de la Independencia para consolidar esa identidad nacional del siglo XIX se hace patente en este análisis sobre el protagonismo de los distintos grupos socio-étnicos en la Independencia de Guayaquil. Acorde a lo propuesto por la Historia Patria, en este análisis se ha podido apreciar que los textos escolares explican la Independencia de Guayaquil como un proyecto de construcción nacional dirigido

39 Cosme J. Gómez Carrasco y Raimundo A. Rodríguez Pérez, "La enseñanza de la Historia y el uso de libros de texto ante los retos del siglo XXI. Entrevista a Rafael Valls Montés”, Historia y Memoria de la Educación, n 6, 2017, p. 364. 
exclusivamente por las élites criollas, cuyo supuesto principal objetivo era conseguir la libertad para toda la Patria. Pese a que hay autores que han denunciado que indigenas, negros, mestizos, zambos, pardos y mulatos obtuvieron pocos beneficios de la Independencia, ${ }^{40}$ todos los textos escolares no dudan en confirmar que gracias a los esfuerzos realizados por las élites criollas de Guayaquil el 9 de octubre de 1820 el Ecuador pudo ser libre.

Después del recuento realizado sobre el protagonismo de los distintos grupos socio-étnicos, a lo largo de este trabajo se ha podido apreciar que el gran protagonismo otorgado a los criollos quienes combaten contra los peninsulares para obtener la libertad, frente a la escasa participación de indigenas, negros, zambos, pardos, mulatos, etc., coincide con los datos propuestos por las investigaciones académicas recientes, donde se manifiesta que la Independencia fue un acontecimiento que enfrentó a criollos versus peninsulares. No obstante, los textos escolares en su labor por lograr la consolidación de esa ansiada identidad nacional no justifican el gran protagonismo de unos y el escaso de otros; ni tampoco el título otorgado a las élites criollas como los héroes y arquitectos de la Patria, así como la catalogación del peninsular como enemigo de la naciente República. Ante este vacío de respuestas, proponemos la vinculación de las nuevas líneas de investigación académica sobre la Independencia a la enseñanza escolar de la Historia por medio de una transposición didáctica del saber sabio (investigaciones académicas actuales), al saber enseñado (textos escolares). Es decir, una transformación de las teorías elaboradas por las investigaciones académicas en un objeto que se pueda enseñar a los educandos, terminando con la utilización de la enseñanza de la Historia para construir una nación y una identidad propias del siglo XIX. ${ }^{41}$

La importancia de esta propuesta radica en que, por medio de esta transformación de las investigaciones académicas en un objeto de enseñanza, se puede someter a debate la interpretación realizada por la Historia Patria del siglo XIX sobre la Guerra de Independencia (presente en los textos escolares) como una contienda que enfrentó a dos naciones e identidades. Los textos escolares no aportan una explicación elaborada sobre el porqué del gran protagonismo de criollos y peninsulares y del escaso protagonismo de indígenas, negros, zambos, etc., dado que el objetivo de esta Historia Patria desde el siglo XIX fue evitar que la Guerra de

40 Holguín y Muñoz son los únicos autores que en un apartado titulado "Consecuencias de la Independencia ecuatoriana", dejan entrever la necesidad de poner en marcha una segunda Independencia en la que verdaderamente se extiendan los ideales de libertad, igualdad y fraternidad a todos los grupos socio-étnicos de los pueblos americanos. No obstante, a pesar de poseer una historiografia que les respalda, estos textos escolares han tenido poca difusión en el ámbito editorial y escolar. Un análisis más detallado sobre esta apreciación se encuentra en: Víctor H. Silva Guijarro, "Republicanos versus absolutistas. Análisis ideológico de la Independencia ecuatoriana en los textos escolares de Historia (19152015). Una propuesta desde el paradigma de la transposición didáctica”, en Manuel Chust (edit.), La Ilusión Heroica. El liberalismo revolucionario en España y América 1820-1823, Editorial Ariadna, Santiago de Chile, 2021, (En prensa).

41 Una explicación más detalla de esta propuesta de transposición didáctica se encuentra en: Víctor H. Silva Guijarro, "Republicanos versus absolutistas. Análisis ideológico de la Independencia ecuatoriana en los textos escolares de Historia (1915-2015). Una propuesta desde el paradigma de la transposición didáctica”, op. cit., (En prensa). 
Independencia sea estudiada como una guerra civil. ${ }^{42}$ Según Pérez Vejo, este objetivo, que se ha mantenido en el tiempo, fue el mismo que se propusieron las élites criollas patriotas tras obtener el triunfo en la Guerra de Independencia. Los líderes criollos a través de los medios de comunicación de la época pusieron en marcha la construcción imaginaria del español como enemigo de la nación, haciéndolo culpable de todas las desigualdades sociales, económicas y étnicas. Por medio de este proceso de hispanofobia, los criollos insurgentes realizaron su propia interpretación de la Independencia como una guerra anticolonial donde el guayaquileño se enfrentó a un supuesto enemigo extranjero: el español. ${ }^{43}$

Este sentimiento de hispanofobia, que forma parte de la Historia Patria del siglo XIX, sigue teniendo mucha presencia tanto en la Historia enseñada (presente en los cien años de textos escolares analizados) como en el discurso político y público del Ecuador. Con esta propuesta de utilización de las investigaciones académicas en el espacio educativo buscamos promover el estudio de la Independencia a nivel escolar tomando como referencia las obras de historiadores como Chust y Frasquet, quienes afirman que la gesta de Independencia sirvió para construir una República blanca y criolla. ${ }^{44} \mathrm{De}$ esta manera, se podría someter a debate la definición de la Independencia realizada por los textos escolares como el origen de una época de libertad para todos los ecuatorianos. Pues una vez consolidadas dichas repúblicas blancas y criollas mediante un proyecto unitario, el protagonismo político, económico y comercial se convirtió en un monopolio exclusivo para los criollos;45 mientras que los indigenas, negros, zambos, mulatos, etc., desaparecieron de los proyectos republicanos del siglo XIX. ${ }^{46}$

Asimismo, esta propuesta se podría convertir en una herramienta para lograr definitivamente el estudio de la Independencia a nivel educativo acorde a los planteamientos de Xavier Guerra ${ }^{47}$ o Jaime Rodríguez, ${ }^{48}$ quienes

42 Cabe recordar que Núñez en su análisis de la Independencia propone que este acontecimiento puede ser estudiado como una guerra civil. El autor justifica la utilización de esta teoría argumentando que durante la guerra de Independencia de Guayaquil tanto el bando realista como el insurgente contó con la presencia de criollos y peninsulares en sus filas. Jorge Núñez Sánchez, Historia del Ecuador, op. cit., p. 10.

43 Tomás Pérez Vejo, "La hispanofobia como elemento de movilización en las guerras de Independencia. ¿Un mito historiográfico?”, en Antolín Sánchez Cuervo y Ambrosio Velasco Gómez (edit.), Filosofía politica de las Independencias latinoamericanas, Biblioteca Nueva, Madrid, 2012, pp. 226-234.

44 Manuel Chust e Ivana Frasquet, Tiempos de Revolución: Comprender Las Independencias Iberoamericanas, Fundación MAPFRE, Madrid, 2013, p. 317.

45 Catalina Reyes, "La fragmentación étnica y politica y su incidencia en la Independencia de la Nueva Granada, 1750-1815”, en Armando Martínez (edit.), Independencia y transición a los Estados Nacionales en los paises andinos: Nuevas perspectivas, Organización de Estados Iberoamericanos, Bogotá, 2005, p. 315.

46 Oscar Almario García, "Racialización, etnicidad y ciudadanía en el Pacífico neogranadino, 1780-1830", en Armando Martínez (edit.), Independencia y transición a los Estados Nacionales en los paises andinos: Nuevas perspectivas, Organización de Estados Iberoamericanos, Bogotá, 2005, pp. 317-352.

47 François-Xavier Guerra, "La ruptura originaria: mutaciones, debates y mitos de la Independencia”, en Germán Carrera Damas; Carole Leal Curiel; Georges Lomné y Frédéric 
definen este acontecimiento como una guerra civil que enfrentó a españoles peninsulares contra españoles americanos; o la tesis propuesta por Pérez Vejo, quien se adhiere a la explicación de la Independencia como una guerra civil, aunque este autor plantea que fue una guerra civil que enfrentó a americanos versus americanos. ${ }^{49} \mathrm{El}$ estudio de la Independencia como una guerra civil, evitado por la Historia Patria, da respuesta tanto al protagonismo principal otorgado por los textos escolares a los criollos y peninsulares, como al escaso protagonismo de indigenas, negros, zambos, etc., pues la Independencia, en contraposición a lo propuesto por los textos escolares, fue un acontecimiento liderado por las élites criollas que aceleró el desarrollo de una revolución política y militar, pero retrasó la consolidación de la igualdad y libertad social para todos los grupos socio-étnicos en las nuevas Repúblicas. ${ }^{50}$

Sin embargo, la propuesta de vinculación de las nuevas lineas de investigación académica sobre la Independencia a la enseñanza escolar de la Historia es un tema complejo, pues ello supondria romper con los imaginarios nacionales que han estado presentes en los textos escolares desde el siglo XIX. Además, de esa forma se dificultaría el afianzamiento del objetivo principal de dichos textos, consistente en la construcción del Estado-nación y la creación de una identidad nacional ideada por las élites criollas en el siglo XIX, en detrimento de la consolidación de una sociedad formada por ciudadanos más analíticos y críticos. ${ }^{51}$ No obstante, en la actualidad esa idea de identidad y de Estado-nación está en peligro por dos motivos; por el proceso de globalización que dificulta mantener unas fronteras nacionales, y por las fuerzas locales y regionales, así como de los diversos grupos étnicos que reclaman un mayor protagonismo en el desarrollo de la vida nacional. ${ }^{52}$ Desde nuestro punto de vista, el problema del concepto de identidad nacional del siglo XIX reside en que es una idea anacrónica que no cumple con las exigencias que demanda la sociedad actual. Cabe recordar que ese Estado-nación surgido de la ruptura del sistema colonial no cumplió con las reivindicaciones de libertad de todos los grupos socio-étnicos, ya que fue un proyecto creado por y para las élites criollas. Ante esta situación, a través de este análisis de los textos escolares (en consonancia con las nuevas líneas de investigación) buscamos promover

Martínez (edit.), Mitos politicos en las sociedades andinas. Orígenes, invenciones y ficciones, Editorial Equinoccio, Caracas, 2006, p. 28.

48 Jaime Rodríguez Ordóñez, "Las Independencias Iberoamericanas en su laberinto", en Manuel Chust (edit.), La Independencias Iberoamericanas en su laberinto. Controversias, cuestiones, interpretaciones, Publicaciones Universidad de Valencia, Valencia, 2010, pp. 311316.

49 Tomás Pérez Vejo, "La hispanofobia como elemento de movilización en las guerras de Independencia. ¿Un mito historiográfico?”, op. cit., pp. 232-235.

50 Oscar Almario García, "Racialización, etnicidad y ciudadanía en el Pacífico neogranadino, 1780-1830”, op. cit., pp. 317-352.

51 Cosme J. Gómez Carrasco y Raimundo A. Rodríguez Pérez, "La enseñanza de la Historia y el uso de libros de texto ante los retos del siglo XXI. Entrevista a Rafael Valls Montés”, op. cit., pp. 317-375.

52 Enrique Ayala Mora, Historiografía ecuatoriana: apuntes para una visión general, op. cit., pp. 19-20. 
la enseñanza de una nueva identidad nacional en la que todas las realidades sociales, étnicas, políticas, económicas, etc., que conforman la actual República del Ecuador tengan cabida. ${ }^{53}$

Pese a las adversidades y gracias a los esfuerzos realizados por las investigaciones académicas, consideramos que el primer paso para superar el arraigo de la Historia Patria del siglo XIX empieza por desarticular una de sus máximas: explicar el pasado desde una perspectiva histórica encerrada en sus propias fronteras nacionales. Esta situación impide levantar una mirada a las demás realidades históricas latinoamericanas. El segundo paso es el reconocimiento de que, sin embargo, la historia ecuatoriana no puede escribirse en clave antinacional porque sigue siendo un país en construcción identitaria, el cual, necesita crear sus propios héroes y mitos para unificar a la ciudadanía. La sociedad ecuatoriana ha entendido que no tiene una identidad, sino varias. ${ }^{54}$ Esta situación debe conducir a una necesaria reforma del concepto de identidad nacional transmitido por los textos escolares mediante esta propuesta de transposición didáctica.

A lo largo de esta investigación se ha podido apreciar que el análisis crítico sobre el proceso de emancipación no es aceptado. Por esta razón, los textos escolares siguen manteniendo la tesis de la Historia Patria sobre la explicación de la Independencia como un acontecimiento supremo que trajo consigo el nacimiento de la República del Ecuador gracias a los esfuerzos de los libertadores. Ante esta situación, consideramos que una de las posibles formas para lograr la reforma de la Historia Patria del siglo XIX se encuentra en ceder el control de la enseñanza de la Historia a los investigadores académicos expertos. A cambio, estos elaborarian una nueva Historia Patria la cual, tomando como referencia la obra de Ayala Mora, tendrá que superar las perspectivas tradicionales mediante un estudio de los diversos proyectos nacionales, asumiendo la creación de un proyecto nacional de futuro ${ }^{55}$ que permita la consolidación de una nueva identidad nacional más inclusiva y articulada a partir de los debates académicos actuales.

Ya para concluir, a través de este nexo entre la Historia enseñada y las investigaciones historiográficas académicas se podría llevar a cabo una remodelación del texto escolar de historia para transformarlo en un instrumento generador de pensamiento y conciencia crítica del pasado; y lograr la difusión a nivel educativo de una explicación de la Independencia desde una perspectiva cultural e integracionista, 56 dejando en un segundo plano la utilización de la Historia Patria del siglo XIX como una herramienta

53 Enrique Ayala Mora, Historiografia ecuatoriana: apuntes para una visión general, op. cit., pp. 19-20.

54 Enrique Ayala Mora, Historiografia ecuatoriana: apuntes para una visión general, op. cit., pp. $20-21$.

55 Enrique Ayala Mora, Historiografia ecuatoriana: apuntes para una visión general, op. cit., p. 21 .

56 Magdalena Cajias de la Vega, "La Independencia frente a la integración latinoamericana en los textos escolares", en Guillermo Bustos y Armando Martínez (edit.), La Independencia en los Paises Andinos: Nuevas perspectivas, Universidad Andina Simón Bolivar / Organización de Estados Iberoamericanos, Bucaramanga, 2004, pp. 252-254. 
nacionalista para llevar a cabo una "ecuatorianización" de los periodos aborigen, colonial e independentista. 57

Enviado el (Submission Date): 23/04/2021

Aceptado el (Acceptance Date): 4/05/2021

57 Guillermo Bustos, El culto a la nación. Escritura de la historia y rituales de la memoria en Ecuador, 1870-1950, Fondo de Cultura Económica / Universidad Andina Simón Bolivar, Quito, 2017, pp. 273-274. 